\title{
Scanning electron microscopy analyses of an ITER plasma-facing unit mockup exposed to extreme ion fluences in Magnum-PSI
}

\author{
M. Balden ${ }^{\mathrm{a}, *}$, S. Elgeti ${ }^{\mathrm{a}}$, T.W. Morgan ${ }^{\mathrm{b}}$, S. Brezinsek ${ }^{\mathrm{c}}$, G. De Temmerman ${ }^{\mathrm{d}}$ \\ ${ }^{a}$ Max-Planck-Institut für Plasmaphysik, Boltzmanstr. 2, D-85748 Garching, Germany \\ ${ }^{b}$ Dutch Institute for Fundamental Energy Research, Eindhoven, The Netherlands \\ ${ }^{c}$ Forschungszentrum Jülich, Institut für Energie- und Klimaforschung-Plasmaphysik, D-52425 Jülich, \\ Germany \\ dITER Organization, Route de Vinon sur Verdon, CS 90 046, 13067 Saint Paul-Lez-Durance, Cedex
}

France

\begin{abstract}
A small-scale tungsten monoblock mockup was exposed to continuous Magnum-PSI plasma beams for 6 different condition: including pure H, D, and He plasmas as well as mixed $\mathrm{D} / \mathrm{He}$ plasmas with low electron temperature $(1.5-5 \mathrm{eV})$, with maximum surface temperatures up to $1600{ }^{\circ} \mathrm{C}$, and ion fluences up to $10^{30} \mathrm{~m}^{-2}$. The recrystallization in the centre of the hottest exposure spots, the absence of cracking on all monoblocks, the formation of a several microns thick nanostructured fuzz layer by pure He exposure, and the presence of various impurities from the exposure in Magnum-PSI on the surface were observed and analysed with a dedicated scanning electron microscope able to handle the entire mockup without any prior cutting.
\end{abstract}

\section{Introduction}

The aim of fusion research is to develop fusion energy as a practical source of energy. The next step on the way to a power plant is the so-called ITER, an experimental device, which is under construction in the frame of a multinational project. Finding a solution for the heat and particle removal from a fusion reactor is a key issue of present-day fusion research. Most of this research is traditionally focused on heat exhaust and influences of high heat loads on the material. Whereas these can be matched routinely in high heat flux test facilities, the ITER divertor components will be additionally exposed to unprecedented ion fluences [1]. Little data is currently available on the effect of high flux, high fluence on the morphology, hydrogen retention, and thermal and mechanical properties of tungsten (W). This is in particular true for the case of actively cooled components, for which the thermal gradient will affect the diffusion into the bulk. Following the installation of superconducting magnetic field coils at Magnum-PSI, it is now possible for the first time to explore the high fluence regime within reasonable machine time of $\sim 20 \mathrm{~h}$ continuous exposure [2, 3].

The current plan for the start of ITER operation is the so-called "staged approach", whereby the machine will progressively be brought up to full power operations. The plan is to have two non-active operational phases, in which hydrogen $(\mathrm{H})$ and helium $(\mathrm{He})$ plasmas will be used to commission the different systems and develop scenarios for the deuterium (D) - tritium (T) operation [4]. Resulting from that, some loading conditions describing the different ITER operational phases were selected and led to an experimental proposal within the EUROfusion consortium [5] to mimic the ITER start-up phase (see Tab. 1). The exposures were performed in Magnum-PSI on a small-scale mockup of the ITER divertor plasma-facing units, consisting of seven tungsten monoblocks brazed on to a $\mathrm{CuCrZr}$ cooling tube. The technical surface as delivered were exposed without any additional surface treatment. Six continuous exposures were performed on different $\mathrm{W}$ monoblocks with pure $\mathrm{H}, \mathrm{D}$, and He plasmas as well as mixed $\mathrm{D} / \mathrm{He}$ plasmas at low electron temperature $(1.5-5 \mathrm{eV})$ with maximum surface temperatures up to $1600{ }^{\circ} \mathrm{C}$, and ion fluences up to $10^{30} \mathrm{~m}^{-2}$, which is of the order of the fluence expected over $\sim 2500$ full power $\mathrm{Q}_{\mathrm{DT}}=10$ discharges $(400 \mathrm{~s})$ [1]. More details about the exposure conditions can be found in [6]. 
In any plasma device such as Magnum-PSI, impurities are always present to some level, which are hard to quantify. For high fluence exposures, it has to be noted that already a very small fraction of, e.g., oxygen $(\mathrm{O})$ and carbon $(\mathrm{C})$ (e.g. $10^{-6}$ ) could lead to significant erosion even of $\mathrm{W}$ in a pure hydrogen plasma (depending on the electron temperature). Furthermore, high loads in the source could lead to an increase in the impurity content in the plasma beam which could result, e.g., in W deposition [7] when a $\mathrm{W}$ electrode is used. Note that copper $(\mathrm{Cu})$ and molybdenum $(\mathrm{Mo})$ are used in the plasma source of Magnum-PSI. In addition, impurities can be generated by erosion of the sample holder (e.g. $\mathrm{Fe} / \mathrm{Cr}$ ), which is also exposed to an intense plasma. The plasma beam might redistribute sputtered material. All this leads to a complicated impurity deposition situation. Nevertheless, this will also happen in a fusion plasma device $[7,8,9]$.

Detailed microscopy analyses can shed light on important questions regarding, e.g., recrystallization, He-induced nanostructured fuzz formation and deposited impurities. To avoid artefacts by sample preparation on surface analyses (especially by cutting) and to select areas of highest interest for further analyses, the entire mockup (as exposed) must be analysed. The scanning electron microscope (SEM) used in the study is dedicated for analysing large and heavy samples with the capability to prepare cross-sections by focused ion beam (FIB) cutting and to perform elemental analyses with X-ray spectroscopy.

This contribution describes the results of the SEM analyses assisted by confocal laser scanning microscopy (CLSM) on the entire mockup with emphasis on: (i) re-crystallization in the centre of the hottest exposed areas, (ii) absence of cracking on all monoblocks, (iii) formation of a nanostructured fuzz layer (several microns thick) due to pure He exposure, and (iv) presence of various impurities on the surface due to exposure in Magnum-PSI.

\section{Experimental}

\subsection{Sample}

The exposed water-cooled small-scale mockup was prepared following the specification for ITER plasma facing units [10]. It was composed of a chain of $7 \mathrm{~W}$ monoblocks $\left(21 \times 28 \times 12 \mathrm{~mm}^{2}\right.$ blocks with $0.5 \mathrm{~mm}$ spacing) produced by Plansee and brazed onto a $\mathrm{CuCrZr}$ tube with a $\mathrm{Cu}$ interlayer. The sample is from the same series used in previous high heat flux studies [11]. The mockup was tested as delivered without any further treatment of the surface beside isopropanol cleaning, i.e., the mockup has a technical surface dominated by grinding grooves with the correlated strong material distortions close to the surface. The microstructural analyses reveal an unusual and non-ITER grade microstructure [6].

\subsection{Magnum exposure}

The quasi continuous plasma exposures were performed in Magnum-PSI (with interruption for realignment of diagnostics and during the night) $[2,3,6]$. Details for the six exposures are given in Tab. 1. The surface temperatures $T_{\text {surf }}$ indicated here are those measured using an infrared camera and a multi-wavelength pyrometer at the centre of the plasma beam (details see [12]) calibrated against a black body radiator [6]. After the first three exposures onto the front side with their beam centre on every other monoblock, the mockup was flipped by $180^{\circ}$ and the last three exposures were performed onto the backside. The exposures on every other monoblock should ensure that the exposures were done on nearly virgin surfaces while performing enough different conditions.

Fig. 1 shows photos of both sides together with marks of the beam centre for the 6 exposures and the analyses positions performed in the frame of the presented study. The different surfaces of the monoblocks are named by F1-7 and B1-7 for the front side and the backside, respectively, and the exposures by the position of the beam centre. The beam width (FWHM) was of the order of 10$15 \mathrm{~mm}$ with a tail leading to features correlated to an exposure with a diameter of about $30 \mathrm{~mm}$, see the halo on B3 and B5 of exposure B4 and on F4 and F6 of exposure F5 (Fig. 1). More details about the exposures can be found in [6]. 


\subsection{Characterisation by microscopy}

The complex conditions of the wall loading in fusion devices by power and particles leads to various surface modifications of the plasma-facing components (PFCs). The clarification of the surface morphology and inner structures in near surface volumes requires detailed microscopy investigations. Non-destructive analyses are mandatory for sequential testing by analysing the same area before and after plasma exposure, i.e., the complete component as installed in the fusion device must fit into the microscope. Therefore, a SEM with FIB (Auriga60 from ZEISS) and analytics, energy and wavelength dispersive X-ray spectroscopy (EDX/WDX from Bruker), was commissioned and put into operation at Max-Planck-Institut für Plasmaphysik. This SEM is equipped with a specially developed heavy-duty stage from Kammrath\&Weiss, which allows to analyse samples up to a mass of $10 \mathrm{~kg}$ with an image resolution of $<5 \mathrm{~nm}$. A sample of $23 \times 10 \times 6 \mathrm{~cm}^{3}$ can be analysed without operational restrictions, i.e., the $X-Y$ stage allows to access completely an area on the sample of $23 \times 10 \mathrm{~cm}^{2}$. The maximal sample size is $44 \mathrm{~cm}$ in length, $27 \mathrm{~cm}$ in width, and $10 \mathrm{~cm}$ without rotation module in height. Cross-sections can be prepared by FIB. A multiple gas injection system enables to deposit W, Pt, or C dominated markers, e.g., for achieving well-prepared cross-sections up to the very surface (e.g., see Figs. 3 and 4) and for erosion measurements. Elemental mapping by EDX/WDX is possible also on cross-sections. Small features can be investigated by using a low energy electron beam (2-5 keV).

The SEM analyses on the entire mockup were performed with this dedicated "Auriga" SEM. The analyses positions of only imaging of the surface, elemental mapping, and imaging of FIB-prepared cross-sections are marked in Fig. 1 as "SEM", "EDX", and "CS", respectively. All presented EDX data were acquired with an electron energy of the SEM beam of $5 \mathrm{keV}$.

In addition, a CLSM (LEXT OLS4000 from Olympus) was used to perform overviews of the surface by optical digital microscopy, to search for possible cracks (and melted surfaces) and to visualize the grain structure on the surface. Furthermore, the achievable height measurements with the laser unit were performed on selected areas. The positions for detailed CLSM analyses are marked in Fig. 1, too.

\section{Results and discussion}

\subsection{Prelude: Base material}

As the surfaces of the mockup were not pre-characterized before the Magnum-PSI exposures, the centre of the surface F1 was taken as reference for an "unexposed" surface. This area is quite far away from the next centre of an exposure beam $(>25 \mathrm{~mm})$ receiving a very low heat load, avoiding any thermally induced change in microstructure of the $\mathrm{W}$ material. Nevertheless, some impurity deposition onto the surface was observed, mainly Mo (see section 3.5).

Fig. 2 shows this area and a cross-section in that region. Clearly, the grinding grooves of the technical surface finishing are visible with a roughness on the $\mu \mathrm{m}$ scale $\left(R_{q}=0.6-1.2 \mu \mathrm{m} ; R_{z}=4-10 \mu \mathrm{m}\right)$. Below the surface, a distorted layer extends over the full observation depth achieved in the performed crosssection of about $20 \mu \mathrm{m}$. The grain size at that depth exceeds easily $10 \mu \mathrm{m}$, leading to observation limitations on FIB-prepared cross-sections. Furthermore, the grains within the top $5 \mu \mathrm{m}$ are strongly distorted, and for the topmost $1 \mu \mathrm{m}$ even very small grains $(<<1 \mu \mathrm{m})$ are present (Fig. 2(b)). More information on the microstructure can be obtained by the analyses on cross-sections after cutting the mockup. From first observations on such cross-sections, a grain size for the base material is of the order of 50-200 $\mu \mathrm{m}$ with some areas in between with grains of $\sim 10 \mu \mathrm{m}[6]$.

The observation of small grains close to the surface and a distorted layers up to the depth of the FIBprepared cross-sections on F1 was confirmed on FIB-prepared cross-sections on B7, B3, F3 and B5 (Fig. 1). The power load on at least the two former surfaces was low. 


\subsection{General surface inspection}

In the inspection of surface with CLSM, the expected absence of cracking (and melting) was confirmed: no cracks (or melt traces) were observed, either in the beam centres or at the monoblock edges (obscured by grinding grooves, which are preserved). Extensive CLSM analyses were performed on the three surfaces exposed at the highest temperatures $\left(>1500^{\circ} \mathrm{C}\right)$ (Fig. 1; F5, B6, B2), where recrystallization could reduce the mechanical properties (see section 3.3). SEM analyses, which were not as extensive as with CLSM regarding the analysed area size, however, with higher resolution, confirmed the absence of cracking (and melting).

\subsection{Recrystallization}

Special emphasis was paid to the question of whether recrystallization took place [11]. The mechanical performance of $\mathrm{W}$ could be degraded by recrystallization. Recrystallized $\mathrm{W}$ is more brittle and, therefore, its enhanced cracking could lead to severe damage of the plasma-facing components. Fig. 3 shows as an example the spot centre of the highest fluence exposure $\left(\sim 10^{30} \mathrm{~m}^{-2}\right)$ with $\mathrm{T}_{\text {surf }}=1580^{\circ} \mathrm{C}$. Note, at this temperature, complete recrystallization occurs for nearly all $\mathrm{W}$ grades [11]. Clearly, recrystallized grains of $10-30 \mu \mathrm{m}$ in size were detected on the surface (also in CLSM, not shown), even if the survived surface topography by the grinding hindered the detection (Fig. 3(a,b)). From CLSM data, the recrystallized grains on the surface extend over an area of roughly $1 \mathrm{~cm}^{2}$ (Fig. 1).

In cross-section (Figs. 3(c-e)), the grain structure is strongly altered up to the full observation depth of 10-15 $\mu \mathrm{m}$ compared to the base material (Fig. 2). The grains are distortion-free and extend beyond the analysable depth on the FIB-prepared cross-sections. These observations are independent of whether the surface was exposed with pure D or with He addition in the plasma if the surface temperature was $\sim 1500{ }^{\circ} \mathrm{C}$. They are in line with results obtained from other devices [11], but contradict to a previous study in Magnum-PSI [11], where no recrystallization by $\mathrm{H}$ plasma exposure at $1500{ }^{\circ} \mathrm{C}$ was observed. Larger depths were analysed via metallographic cross-sections and an assessment of the recrystallization temperature under these loading conditions using finite-element-method analysis to map the temperature across and inside the monoblock can be found in [6].

\subsection{Surface morphology changes by He}

Clearly, the pure He exposure changed the surface morphology as was already observable by naked eye: the exposed area became blackened (B4, Figs. 1(b) and 4(a)). In SEM, typical He-induced nanostructured fuzz was observed at $\mathrm{T}_{\text {surf }} \sim 1000{ }^{\circ} \mathrm{C}$ (Fig. 4) despite the rather low ion energy $(<18 \mathrm{eV}$ due to floating conditions [6]), which is at the lower energy limit for fuzz formation [13-15]. Note that Mo is incorporated in the fuzz (see below; Fig. 4(e)). Addition of He as for the exposures of blocks F7 and B6 with mixture D+He did not lead to fuzz formation, despite that has been found for mixed $\mathrm{H}+\mathrm{He}$ exposure [16, 17]. Probably the observed He flux threshold depends on the He energy [17], which was for these two exposures lower than for the pure He one [6]. Unfortunately, only the gas mixture and not the ratio $\mathrm{He} / \mathrm{D}$ flux is known. Note the $\mathrm{W}$ surface structure of the mixed exposures (F7 and B6) is not distinguishable from that one of the pure D exposures at the same surface temperature of $\sim 1500{ }^{\circ} \mathrm{C}$ ( $\mathrm{F} 5$ and $\mathrm{B} 2$ ) and that this temperature is still in temperature range for fuzz formation for $\mathrm{W}[13,14]$; see section 3.3.

The thickness of the fuzz layer across the blackish spot was analysed by a series of FIB-prepared cross-sections (Figs. 1(b) and 4(a)). Interestingly, the layer is thinner in the centre of the spot $(\sim 3 \mu \mathrm{m})$ than at the edge $(\sim 8 \mu \mathrm{m})$ (Fig. 4(c)), even if the structure of the fuzz on the surface and in the crosssection is the same. Remarkable is that the ion energy at the edge was lower than in the centre [6], i.e, further below the reported threshold [13-15].

In addition, the fuzz did not form anymore a continuous layer at the edge of the spot (about $7 \mathrm{~mm}$ from the beam centre with surface temperature $>750{ }^{\circ} \mathrm{C}$ ). These structures can be described as fuzz balls (Fig. 4(f)). Possibly, they have a similar origin as the reported nano-tendril bundles [18, 19], 
which grow as isolated filigrane structures with high height-to-lateral size ratio on the $\mathrm{W}$ surface under various conditions. The origin of the nano-tendril bundles is still unclear. As possible explanations, e.g., the modulation of He energy [19] and the impurity content in the He plasma are discussed [18].

As the neighbouring monoblock edge across a gap received nearly the same impacting particle fluxes, but the total power deposited on each block was strongly different, the surface temperature differed strongly between these two edge regions (Figs. $4(\mathrm{~d}, \mathrm{~h})$ ). In the case from block B4 to B5, this led to a temperature below $700{ }^{\circ} \mathrm{C}$ on B5, so that the conditions for fuzz formation were not fulfilled, i.e., no blackened area on block B5 was observed (Fig. 4(a)). Nevertheless, at the edge of block B5 a very porous layer with apparent closed pores was formed with a thickness of $\sim 0.5 \mu \mathrm{m}$ (Fig. 4(h)). The porous layer is also present in between and beneath the fuzz balls at the edge of the blackish area on B4 (not shown), but not where the fuzz layer was completely formed (Fig. 4(c,d)). The surface of this porous layer has some edgy topography, as shown in Fig. 4(g)).

In addition, the fuzz layer seems to consist of two sub-layers with higher Mo content in the buried one (see e.g. Fig. 4(e)). The Mo seems to be incorporated into the branches of the fuzz. The ratio W to Mo resulting from the quantification of the EDX data for the top layer and deeper layer in Fig. 4(e)) is 8 and 3, respectively. The ratio shows a strong variation between the different cross-sections (Fig. 4(a)) reaching even values below 1. This sub-layer structure and the incorporation reflect the transport of the $\mathrm{W}$ from the sub-surface $(<100 \mathrm{~nm})$ to the surface, i.e. intermixing, as observed by [20]. Probably some changes during the Magnum-PSI exposure, e.g. the interruption, caused the substructure. It is not known which effect the Mo has on the fuzz formation and growth. Fuzz formation is also reported for pure Mo [21]. The temperature required for Mo fuzz formation $\left(530-1200{ }^{\circ} \mathrm{C}\right)$ is lower than for $\mathrm{W}\left(730-1730{ }^{\circ} \mathrm{C}\right)[14,21,22]$. Furthermore, the growth of fuzz could also be promoted by the deposition of the Mo, similar to the observations in [23].

Unfortunately, due to the interplay of many parameters needed for fuzz formation, which are in the frame of this experiment not well known, no definite explanation and conclusion can be drawn. Nevertheless, remarkable is the formation of fuzz despite the low He energy, and it can be speculated that the incubation fluence scales with the He energy.

\subsection{Impurities}

\subsubsection{Prelude}

Some remarkable observations were made in correlation to impurities deposited from the plasma beam (see next subsections). Molybdenum (Mo), copper $(\mathrm{Cu})$, iron $(\mathrm{Fe})$, chromium $(\mathrm{Cr})$ and tin $(\mathrm{Sn})$ were observed via optical emission spectroscopy in the plasma beam [6]. Note that the detected impurity amounts on the surfaces are small compared to the acquired fluences as illustrated by the following assessment. Layers of about $100 \mathrm{~nm}$ contain about $10^{22} \mathrm{at} / \mathrm{m}^{2}$. If a sticking probability of 1 is assumed, which is usual for metals, and re-erosion is neglected, a fraction of $\sim 10^{-8}$, i.e., $<<10^{-6}$ for impurities in the plasma beam can be estimated to create such a layer.

\subsubsection{Tin}

On top of the issues of impurities originated by the plasma generation as mentioned in sections 1 and 3.5.1, in previous exposure campaigns of Magnum-PSI, a quite substantial amount of tin ( $\mathrm{Sn}$ ) was introduced into the chamber [24]. Unfortunately, this Sn seemed to be redistributed and deposited onto the mockup. Sn was detected in nearly all EDX analyses on the surface of the W monoblocks. Sn structures as observed in dedicated Sn plasma exposure experiment in another plasma device [25] were found on the mockup. These structures are Sn sponge-like structures with bubbles in the Sn branches (as observed for exposure just below the melting point of $232{ }^{\circ} \mathrm{C}$ [25]) and Sn droplets with a contact angle of only about $45^{\circ}$. In particular, they were observed on the sidewall in the gaps between the W monoblocks, e.g., Sn droplets on F4 \& F5 (diameter of 10-50 $\mu$ m) and Sn sponge on F6 (branch thickness of $\sim 3 \mu \mathrm{m}$, branch length of $>50 \mu \mathrm{m}$ ). In some cases, $\mathrm{Sn}$ was found incorporated in deposited structures and layers containing large fractions of $\mathrm{Mo}, \mathrm{Fe}$, and $\mathrm{Cu}$. 


\subsubsection{Molybdenum}

On all areas around the plasma beam centre (Figs. 3(b,d,e), 4(e)), but also far away (Fig. 2), Mo was observed. At some locations, the thickness of Mo-dominated layers was determined on FIB-prepared cross-sections, which is in the range of $50 \mathrm{~nm}$ (maximal observed of $100 \mathrm{~nm}$ ). Note that a systematic analysis of the thickness across the samples was not performed.

Interestingly, the Mo distribution varies laterally (tens of $\mu \mathrm{m}$ ) in areas showing large recrystallized grains on the surface. In elemental maps of the surface obtained by EDX, the grain structure is visible, i.e., the amount of Mo varies from grain to grain (Fig. 3(b)). As these regions exhibited high temperatures, the mobility of Mo (and $\mathrm{Sn}$ ) across the surface varied with grain orientation. In addition, the Mo diffusion along grain boundaries was enhanced compared to diffusion into grains as can be concluded from the observation of Mo-decorated grain boundaries observed on FIB-prepared cross-sections (Fig. 3(d,e)). The Mo enrichment at the grain boundaries fades out at a depth of 2$3 \mu \mathrm{m}$.

The nanostructured fuzz observed on block B4, contains also a large fraction of Mo (Fig. 4(e)). It seems that the Mo is incorporated into the branches of the fuzz (see section 3.4).

\subsubsection{Copper and iron/chromium}

$\mathrm{Cu}$ and $\mathrm{Fe}$ together with $\mathrm{Cr}$ are often significantly present on the surface. They sometimes even dominate the observed impurities. Traces of $\mathrm{Cu}$ and Fe were very frequently observed on all surfaces.

The dark areas at the edges of block B3 to block B2 are attributed to a layer dominated by Cu. Note that the exposure on B2 was the last one being performed (Tab. 1). From the lateral distribution of this $\mathrm{Cu}$ on the exposed surface, it could be speculated that the $\mathrm{Cu}$ originated from the $\mathrm{CuZrCr}$ tube, e.g., by sputtering. This is supported by the observation that the $\mathrm{Cu}$ amount on the side of block $\mathrm{B} 3$ in the gap increases with reducing distance to the tube. On the other hand, the temperature gradient may originates this variation, and the origin of the $\mathrm{Cu}$ is the plasma source, which is also very reasonable.

The analyses of the blue-coloured area in Fig. 1 on F2 show a Fe+Cr dominated layer. This large amount of $\mathrm{Fe}+\mathrm{Cr}$ extents across the gap onto F3. In the centre of the exposure on F3, a double layer is observed, i.e., a $>50 \mathrm{~nm}$ Mo-dominated layer is covered by a $<50 \mathrm{~nm} \mathrm{Fe}+\mathrm{Cr}$-dominated layer. This leads to the conclusion that the $\mathrm{Fe}+\mathrm{Cr}$ producing event was after the exposure on $\mathrm{F} 3$, which was the first one of all exposures (Tab. 1).

\section{Conclusion and Summary}

A small-scale mockup of the ITER divertor plasma-facing units, consisting of seven tungsten monoblocks brazed on to a $\mathrm{CuCrZr}$ cooling tube, was exposed to a variety of high fluence, high flux plasma loads in Magnum-PSI [2, 3, 6]. Six exposures were performed including H, He, D and D:He (95:5) plasmas with low electron temperature $(1.5-5 \mathrm{eV})$ and high electron density $\left(1 \times 10^{20}-2 \times 10^{21} \mathrm{~m}^{-}\right.$ ${ }^{3}$ ), resulting in a maximum surface temperature in the range $750-1600{ }^{\circ} \mathrm{C}$ (Tab. 1) [6]. These exposures aimed to closely replicate the partially detached conditions expected at the divertor strike lines during the different operational phases of the ITER staged approach [4]. The largest fluence, $10^{30} \mathrm{D} \mathrm{m}^{-2}$, achieved in 19 hours, is equivalent to around one year of full power operation, i.e., a few thousand discharges of $400 \mathrm{~s}$ duration [1].

The first step of the post-mortem analysis, with SEM, EDX and CLSM, is presented and aimed at studying how such long-term exposures affected the microstructure of each monoblock. The monoblocks behaved as expected. No cracking (or melting) was observed. When the maximal surface temperature exceeded $1500^{\circ} \mathrm{C}$, as was the case in four exposures, recrystallization was observed. No strong erosion took place, as the survival of the grinding grooves even in the recrystallized areas demonstrates. Furthermore, only the pure He exposure led to surface topography changes of the 
surface layer at $\sim 1000{ }^{\circ} \mathrm{C}$. The well-studied nanostructured fuzz was formed, despite the rather low ion energy $(<18 \mathrm{eV})$ [13-23]. The influence of the Mo presence on the fuzz formation is unknown. The addition of He to the $\mathrm{D}$ plasma at $\sim 1500^{\circ} \mathrm{C}$ did not lead to such surface changes; only recrystallization occurred.

The presence of impurities implies some complications for the analyses. Nevertheless, the impurities led to some unexpected observations, e.g., the enhanced diffusion of Mo along the grain boundaries in the recrystallized areas and the sponge-like Sn structures [25]. Overall, the amount of impurities arriving with the plasma is small related to the high accumulated fluences, and the impurities do not harm the positive performance for the different high fluence exposure conditions, i.e., these experiments do not emphasize any additional concerns for the different exposure conditions in the ITER staged approach. At these high fluence exposures on a small-scale ITER plasma facing unit mockup, no catastrophic behaviour appeared.

\section{Acknowledgment}

The actively-cooled monoblock chain was supplied by the ITER Organization and F4E as part of a EUROfusion-funded experiment to investigate the effect of ITER-relevant fluences on the properties of tungsten. This work has been carried out within the framework of the EUROfusion Consortium and has received funding from the Euratom research and training programme 2014-2018 and 2019-2020 under grant agreement No 633053. Work partly performed under EUROfusion WP PFC. The views and opinions expressed herein do not necessarily reflect those of the European Commission or of the ITER Organization.

\section{References}

[1] De Temmerman G, Hirai T, Pitts RA 2018 Plasma Phys. Control. Fusion 60044018.

[2] van Eck HJN et al. 2019 Fusion Eng. Des. 142 26-32

[3] van de Pol et al. 2018 Fusion Eng. Des. 136 597-601

[4] ITER Organisation 2018 Research Plan within the Staged Approach ITER Technical Report ITR-18-003 https://www.iter.org/technical-reports

[5] Brezinsek S et al. 2017 Nuclear Fusion 57116041

[6] Morgan TW, Schwarz-Selinger T, Balden M, Li Y, Rosas Saad JA, Loewenhoff T, Wirtz M, Sietsma J, Brezinsek S, De Temmerman G 2019 ITER monoblock performance under lifetime loading conditions in Magnum-PSI, Presented at $17^{\text {th }}$ PFMC, Eindhoven, May 2019, submitted to Physica Scripta

[7] Balden M, Lindig S, Manhard M, Krieger K, ASDEX Upgrade Team 2013 J. Nucl. Mater. 438 S220-3

[8] Mayer M, Likonen J, Coad JP, Maier H, Balden M, Lindig S, Vainonen-Ahlgren E, Philipps V, JET-EFDA Contributors 2007 J. Nucl. Mater. 363-365 101-106

[9] Kallenbach A et al, 2011 J. Nucl. Mater. 415 S19-26

[10] Hirai T et al. 2015 J. Nucl. Mater. 463 1248-51

[11] Loewenhoff T et al. 2015 Nuclear Fus. $\mathbf{5 5} 123004$

[12] van den Berg MA et al. 2013 J. Nucl. Mater. 438 S432-4

[13] Kajita S, Sakaguchi W, Ohno N, Yoshida N, Saeki T 2009 Nucl. Fusion 49095005

[14] Kajita S et al. 2018 Surf. Coat. Technol. 430 86-92

[15] Takamura S, Ohno N, Nishijima D, Kajita S 2006 Plasma Fus. Res. 1051 
[16] Greuner H et al. 2014 J. Nucl. Mater. 455 681-4

[17] Baldwin, MJ, Doerner RP, Nishijima D, Tokunaga K, Ueda Y 2009 J. Nucl. Mater. 390-391 886-890

[18] Hwangbo D, Kajita S, Tanaka H, Ohno N 2019 Nucl. Mater. Energy 18 250-7

[19] Woller KB, Whyte DG, Wright GM 2017 Nucl. Mater. Energy 12 1282-7

[20] Doerner RP, Nishijima D, Krasheninnikov SI, Schwarz-Selinger T, Zach M 2018 Nucl. Fusion 58066005

[21] Takamura S 2014 Plasma Fus. Res. 91405131

[22] De Temmerman G et al. 2012 J. Vac. Sci. Technol. A 30041306

[23] Kajita S, Kawaguchi S, Yoshida N, Ohno N, Tanaka H 2018 Nucl. Fusion 58106002

[24] Morgan TW et al. 2018 Plasma Phys. Control. Fusion 60014025

[25] Manhard M, Schwarz-Selinger T, Balden M, Dürbeck T, Maier H, Neu R 2019 Erosion and Deuterium Uptake of Solid and Liquid Tin in a Low-Temperature Deuterium Plasma,

Presented at $17^{\text {th }}$ PFMC, Eindhoven, May 2019, submission to Nuclear Fusion in preparation

\section{Table}

Tab. 1: Exposure conditions in Magnum-PSI onto the small-scale ITER W monoblock mockup. The max values are valid for the beam centre. The electron temperature and density were in the range 1.5$5 \mathrm{eV}$ and $1-20 \times 10^{20} \mathrm{~m}^{-3}$. The ratio of gas flow rates of $\mathrm{D}$ and He was 95:5.

\begin{tabular}{|c|c|c|c|c|c|c|c|c|c|}
\hline$\frac{y}{\ddot{0}}$ & 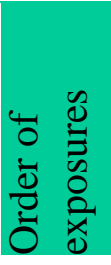 & 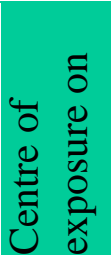 & 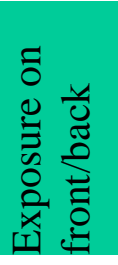 & $\begin{array}{l}\tilde{U} \\
\frac{\tilde{U}}{0} \\
\tilde{0} \\
\tilde{n} \\
\tilde{\Xi} \\
\tilde{\Xi} \\
\frac{\pi}{0}\end{array}$ & 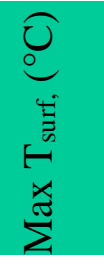 & 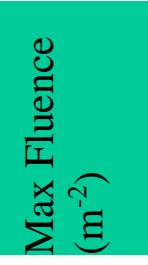 & 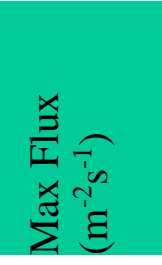 & 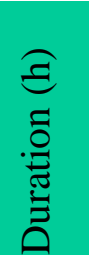 & 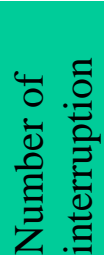 \\
\hline 1 & & & None & & & & & & \\
\hline 2 & 6 & B2 & Back & D & 1570 & $4.1 \times 10^{29}$ & $1.0 \times 10^{25}$ & 11.2 & 2 \\
\hline 3 & 1 & F3 & Front & $\mathrm{H}$ & 750 & $1.0 \times 10^{29}$ & $1.2 \times 10^{24}$ & 22.6 & 2 \\
\hline 4 & 5 & B4 & Back & $\mathrm{He}$ & 1050 & $2.5 \times 10^{28}$ & $2.9 \times 10^{24}$ & 2.9 & 1 \\
\hline 5 & 2 & F5 & Front & D & 1580 & $1.0 \times 10^{30}$ & $1.4 \times 10^{25}$ & 19.7 & 7 \\
\hline 6 & 4 & B6 & Back & D:He (95:5) & 1555 & $5.0 \times 10^{29}$ & $1.3 \times 10^{25}$ & 17.8 & 1 \\
\hline 7 & 3 & F7 & Front & $\mathrm{D}: \mathrm{He}(95: 5)$ & 1575 & $2.8 \times 10^{29}$ & $1.2 \times 10^{25}$ & 6.5 & 0 \\
\hline
\end{tabular}




\section{Figures}

Fig. 1: Photos of the two plasma-exposed faces, (a) "Front" and (b) "Back", of the small-scale ITER W monoblock mockup. The centre of the plasma beams, i.e., the position of max $\mathrm{T}_{\text {surf }}$ in Tab. 1, and the analyses positions are marked (imaging of surface: SEM; imaging of FIB-prepared cross-sections: CS; elemental mapping: EDX; detailed digital microscopy with roughness measurement: CLSM). Note, for the CLSM data points, blue indicates unchanged areas, orange marks recrystallized areas and yellow labelled areas showing already strong deviation from unchanged areas, but are not clearly recrystallized.

Fig. 2: (a) top view SEM image of an "unexposed" area, i.e., the centre of F1, showing the grinding grooves of the technical surface of the monoblocks and (b) a FIB-prepared cross-section illustrating the zone with small and distorted $\mathrm{W}$ grains below the surface (position of cross-section marked in (a) as dotted line). The elemental composition of the thin black line visible in the cross-section (b) was determined by EDX as dominated by Mo. Note the cross-section is viewed under an angle of $36^{\circ}$, and an artificial Pt-C coating is present above the black line of Mo (b).

Fig. 3: (a) top view SEM image of the beam centre of exposure $\mathrm{F} 5,10^{30} \mathrm{D} / \mathrm{m}^{2}$ at $\mathrm{T}_{\text {surf }}$ of $1580{ }^{\circ} \mathrm{C}$ visualizing large recrystallized grains. (b) elemental intensity maps (EDX) of W, Mo and Sn of the region of (a) illustrating the grain dependent decoration of $\mathrm{W}$ grains by the deposited impurities. (c) FIB-prepared cross-section, which is shown as a scaled overlay in (a), too, and its position is marked by a white line in (a,b). (d) and (e) magnified parts of the region left and in the middle of (c), respectively. As inserts, the elemental intensity maps of a part of the areas shown in (d) and (e) clearly indicate the decoration of the grain boundaries with Mo and a Mo-dominated layer of $50 \mathrm{~nm}$ on the surface. Note the cross-section is viewed under an angle of $36^{\circ}$, an artificial Pt-C coating is present above the black line of Mo (c-e), and EDX data were acquired with $5 \mathrm{keV}$ electron energy.

Fig. 4: (a) photo of the spot of exposure B4, $2.5 \times 10^{28} \mathrm{He} / \mathrm{m}^{2}$ at $1050{ }^{\circ} \mathrm{C}$ with labelling of analysed positions; (b) top view SEM image of beam centre showing the filigrane tendrils of the fuzz; (c) SEM image of the cross-section at the edge of the beam; (d) SEM image of the cross-section very close to the edge of monoblock B4 (see (a)), but also close to the beam centre; (e) elemental intensity maps of the region of (d); (f) top view SEM image of the edge of the spot with "fuzz-balls" of $\sim 10 \mu \mathrm{m}$ height and $\sim 20-30 \mu$ m diameter; (g) top view SEM image of the region between the "fuzz balls" (position marked in (f); (h) SEM image of the cross-section very close to the edge of monoblock B5 (distance to cross-section shown in $(\mathrm{d}, \mathrm{e})$ is only $0.8 \mathrm{~mm})$. Note the cross-sections are viewed under an angle of $36^{\circ}$, an artificial Pt-C coating is present above the fuzz layer (c-e,h), and EDX data were acquired with $5 \mathrm{keV}$ electron energy. 

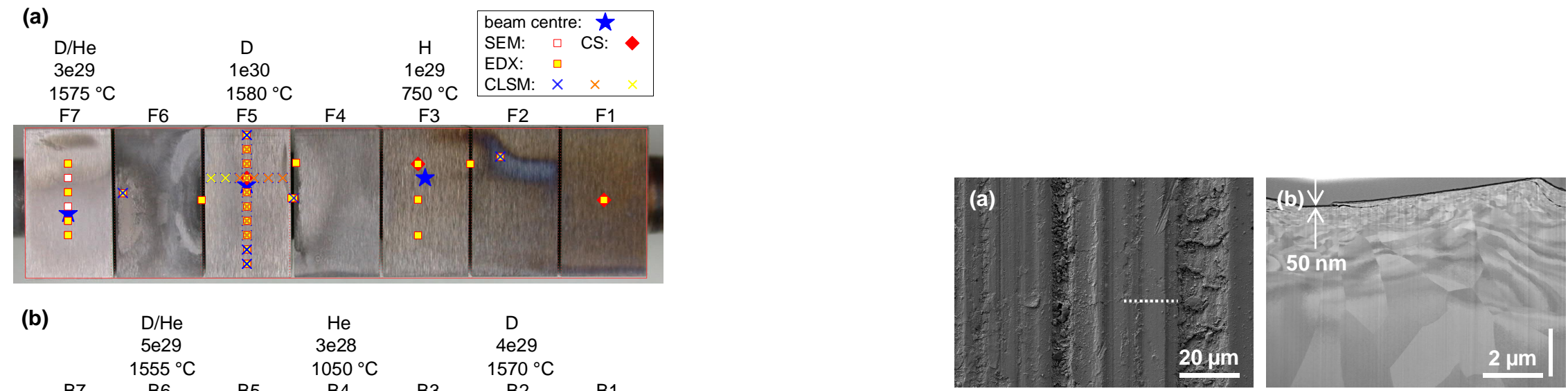

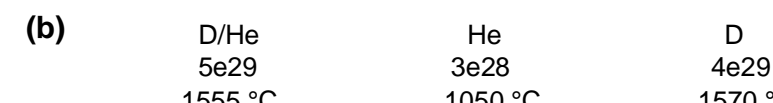

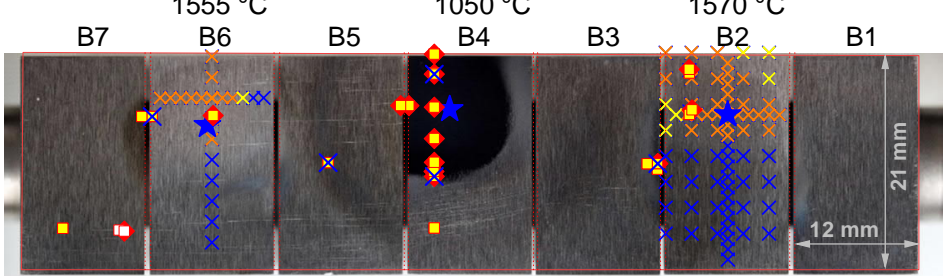

Fig. 2

Fig. 1 


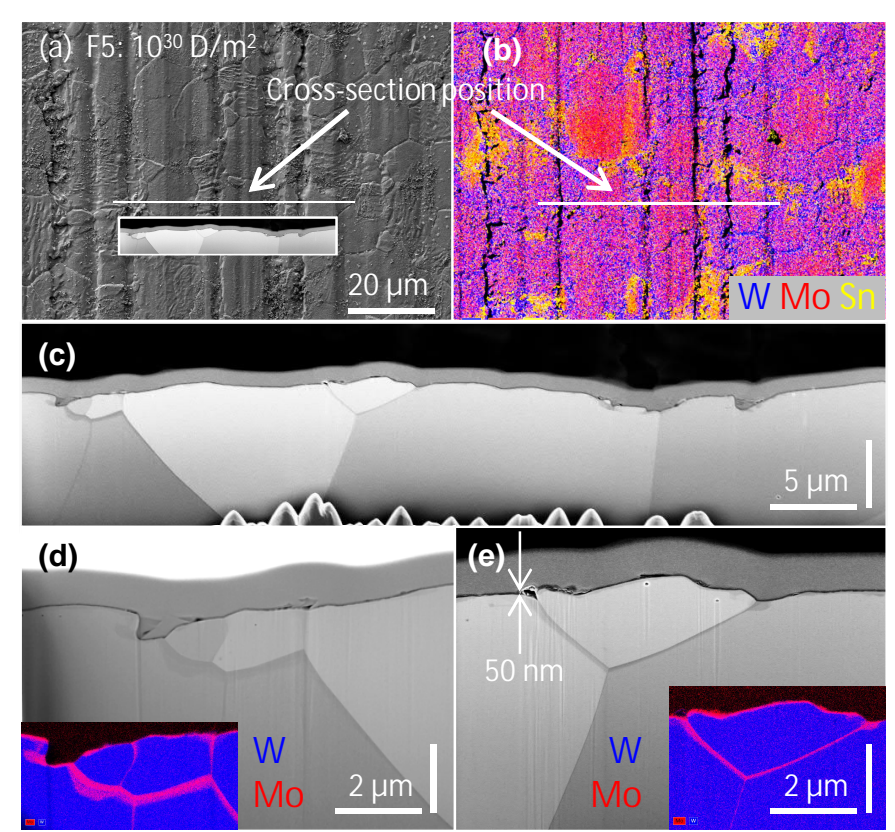

Fig. 3
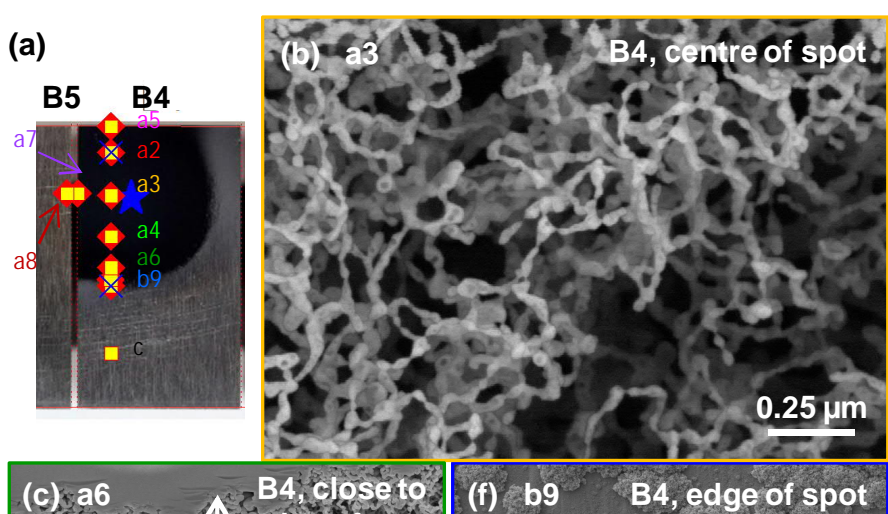

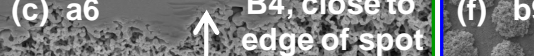

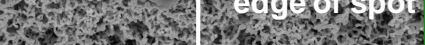

i.

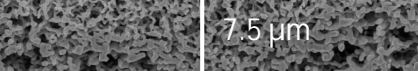

7.

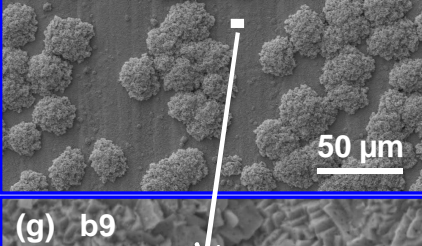

(d) $=$ a7 close to edge of B4

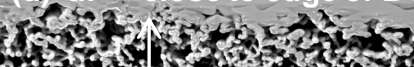

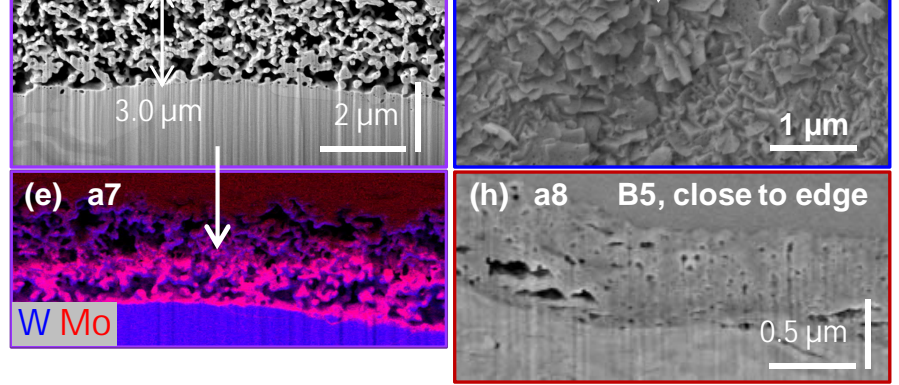

W Mo 